\title{
Crack Tip Plastic Zone Formation during Pipeline Hydrostatic Testing
}

Jian Li, M.Elboujdaini and P.Liu

Materials Technology Laboratory, CANMET, 568 Booth St. Ottawa, Ontario, Canada, K1A 0G1

Concerns about stress-corrosion cracking (SCC) in the pipeline industry have increased in the past years due to an increase in the frequency of pipeline failures. The SCC-related failures have occurred not only in natural gas pipelines but also in pipelines transporting oil. In 2003, the US Department of Transportation issued an advisory notice to all US pipeline owners and operators to assess their pipeline SCC risk in both high-pH and low-pH environments [1]. Pipeline SCC inspections are carried out either by in-line inspection using shear wave ultrasonic crack detection (USCD) or, most effectively, by hydrostatic testing, which has been used in the oil and gas pipeline industry for decades. In a typical hydrostatic test, existing pipelines are pressurized using water up to $110 \%$ of the materials' specified minimum yield strength (SMYS) and hold for a designated length of time. The hydrostatic test is very effective in detecting near-critical cracks [2]. However, there are concerns that sub-critical sized cracks may grow larger and some blunt dormant cracks may be re-activated during hydrostatic tests.

A section of in-service pipeline containing a colony of SCC cracks was investigated; hydrostatic tests were performed in 1994 and after in 2004. A small sample containing SCC cracks was cut out, polished and finished with $0.05 \mu \mathrm{m}$ colloidal silica using a special sample-preparation routine suitable for imaging using a focused-ion beam (FIB) microscope. In recent years, FIB microscopes have found many applications in microstructural characterization. The FIB secondary-electron (SE) images provide enhanced crystallographic contrast [3]. Misorientations as small as $2^{\circ}$ can be detected in FIB SE images, making it an ideal tool to detect local plastic deformation in pipeline materials.

Figure 1 shows a typical FIB image taken at an SCC crack-tip from a sample that had not been subjected to hydrostatic testing. The crystallographic orientation contrast indicated no apparent plastic zone ahead of the crack tip. Figure 2 shows a crack tip from a pipe subjected to hydrostatic tests; this sample shows a plastic zone near the crack tip. The existence of deformed substrate near crack tips could promote further SCC propagation. Figure 3 shows a montage of a section of a long crack. The top portion of this crack is much wider and transformed to a distinctively thinner crack. Apparent plastic zones exist at both the transition zone and the thin crack tip. It can be deduced that the thicker SCC existed prior to the hydrostatic test in 1994 and became inactive for a period of time (dormant crack before 1994 hydrostatic testing). Signs of hydrotest in 1994 are marked by the plastic deformation in the transition zone. This SCC became re-activated and started to propagate again after the 1994 hydrostatic test, and the 2004 hydrostatic test created the second plastic zone at the crack tip.

The FIB SE imaging provides new insights into the effect of hydrostatic tests on steel pipelines. The current microstructural features indicate that caution should be exercised when planning for hydrostatic testing since it may re-activate some inactive SCC. These plastic zones are not detectable by either optical microscope or SEM. A detailed study will be published elsewhere.

\section{REFERENCES}

[1]. Office of Pipeline Safety, "SCC Threat Advisory Notice", October 2003. 
[2]. B.N. Leis, R.D., Galliher, R.L., Sutherby and R. Sahney, Proceedings of the IPC 2004, October 4-8, 2004, Calgary, Alberta, Canada, Paper No: IPC04-0665.

[3]. Jian Li, M. Phaneuf and S. Saimoto, Steps Towards Orientation Measurement in a Focused Ion Beam Microscope, Conference of Microscopy Society of Canada, 1998, Ottawa, Ontario.

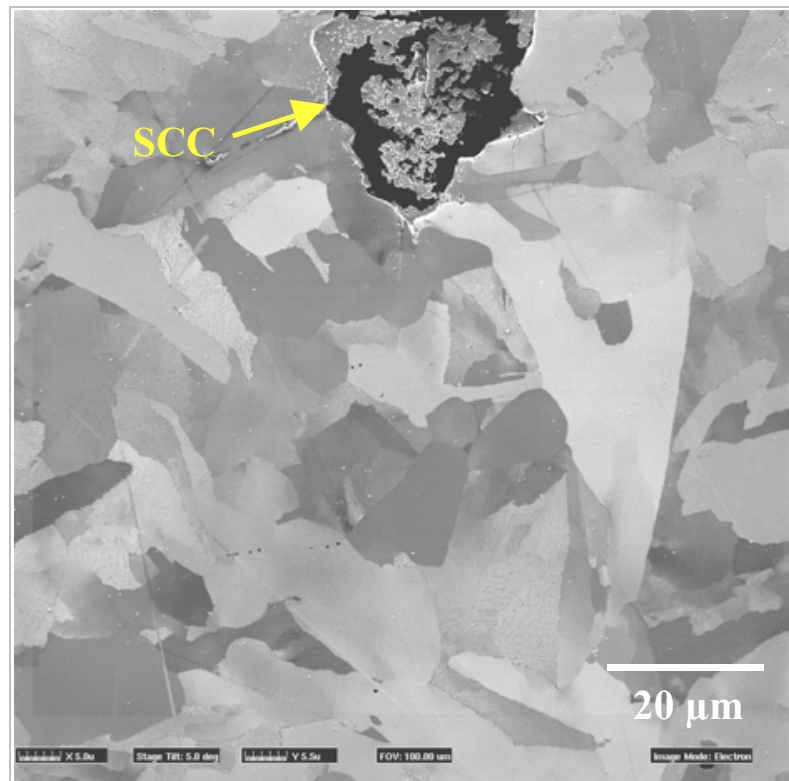

Figure 1. SCC tip from a sample with no hydrostatic test.

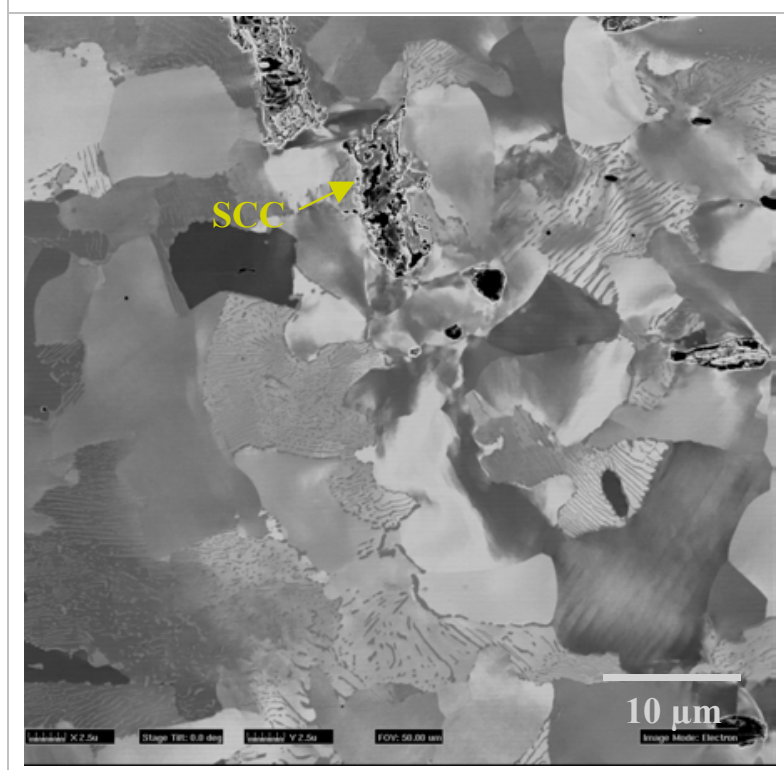

Figure 2. Plastic zone at a large SCC tip resulting from hydrostatic testing.

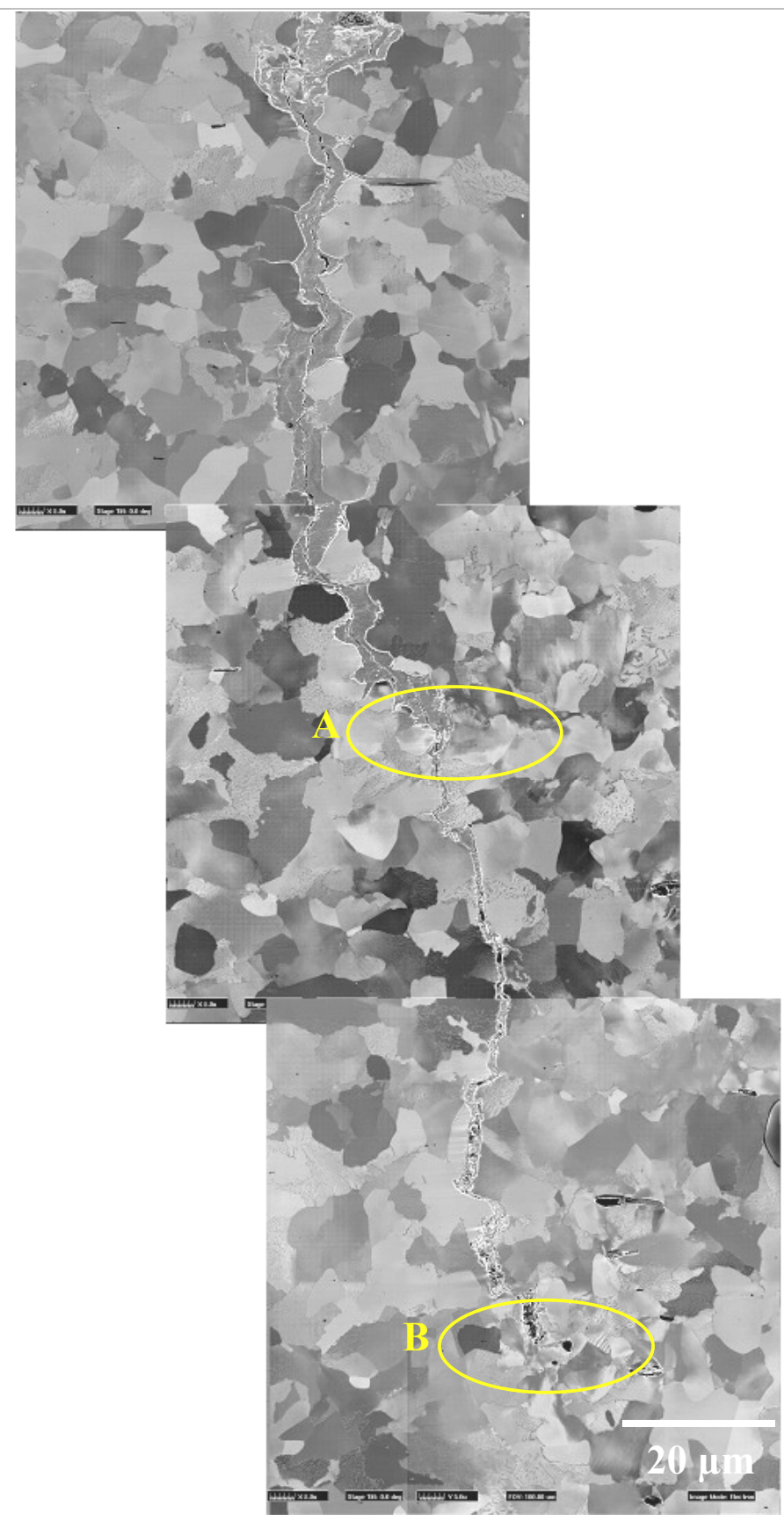

Figure 3. Montage image of a large SCC showing the SCC re-activation and propagation from the earlier hydrostatic test. A: Plastic zone at the SCC tip due to 1994 hydrotest, B: SCC tip and plastic zone resulting from the hydrotest in 2004. 